\title{
INTEGRATION OF LIDAR DATA AND ORTHOIMAGE FOR AUTOMATIC 3D BUILDING ROOF PLANE EXTRACTION
}

\author{
Mohammad Awrangjeb ${ }^{a}$, Clive S. Fraser ${ }^{b}$ and Guojun Lu $^{a}$ \\ ${ }^{a}$ Gippsland School of Information Technology, Vic 3842 Australia \\ Phone: +61 3990 26462, E-mail: \{Mohammad.Awrangjeb, Guojun.Lu\}@monash.edu \\ ${ }^{b} \mathrm{CRC}$ for Spatial Information, Dept. of Infrastructure Engineering, University of Melbourne, Australia \\ Phone: +61 38344 4117, E-mail: c.fraser@ unimelb.edu.au
}

\begin{abstract}
Automatic 3D extraction of building roofs from remotely sensed data is important for many applications including city modeling. This paper proposes a new method for automatic 3D roof extraction through an effective integration of LIDAR (Light Detection And Ranging) data and multispectral orthoimagery. Using the ground height from a DEM (Digital Elevation Model), the raw LIDAR points are separated into two groups. The first group contains the ground points that are exploited to constitute a 'ground mask'. The second group contains the non-ground points which are segmented using an innovative image line guided segmentation technique to extract the roof planes. The image lines extracted from the grey-scale version of the orthoimage are classified into several classes such as 'ground', 'tree', 'roof edge' and 'roof ridge' using the ground mask and colour and texture information from the orthoimagery. During roof plane extraction the lines from the later two classes are used to fit roof planes to the neighbouring non-ground LIDAR points. Finally, a new rule-based procedure is applied to remove planes constructed on trees. Experimental results show that the proposed method successfully removes vegetation and offers high extraction rates.
\end{abstract}

Index Terms- Building, feature, extraction, reconstruction, automation, integration, LIDAR, orthoimage

\section{INTRODUCTION}

Up to date 3D building models are important for many GIS (Geographic Information System) applications such as urban planning, and disaster management. Therefore, 3D building reconstruction has been an area of active research within the photogrammetric, remote sensing and computer vision communities for the last two decades. Building reconstruction implies the extraction of 3D building information, which includes corners, edges and planes of the building facades and roofs from remotely sensed data such as photogrammetric imagery and height data. Digital reconstruction of the facades and roofs then follows using the available information. This research concentrates on $3 \mathrm{D}$ extraction of roof planes. Although the problem is well understood and in many cases accurate modelling results are delivered, the major drawback is that the current level of automation is comparatively low [1].

$3 \mathrm{D}$ building roof reconstruction from aerial imagery seriously lacks in automation partially due to shadows, occlusions and poor contrast. In addition, the extracted information invariably has low vertical accuracy. Fortunately, the introduction of LIDAR (Light Detection And Ranging) has offered a favourable option for improving the level of automation in 3D reconstruction when compared to image-based reconstruction alone. However, the quality of the reconstructed building roof from the LIDAR data is restricted by the ground resolution of the LIDAR which is still generally lower than that of the aerial imagery. That is why the integration of aerial imagery and LIDAR data has been considered complementary in automatic 3D reconstruction of building roofs. However, the question of how to effectively integrate the two data sources with dissimilar characteristics still arises; few approaches with technical details have thus far been published.

The 3D reconstruction of building roofs comprises two important steps [2]. The detection step is a classification task and delivers regions of interest in the form of $2 \mathrm{D}$ lines or positions of the building boundary. The reconstruction step constructs the $3 \mathrm{D}$ models within the regions of interest using the available information from the sensor data. The detection step significantly reduces the search space for the reconstruction step. In this section, a review of some of the prominent methods for 3D roof reconstruction is presented.

Methods using ground plans [3] simplify the problem by partitioning the given plan and finding the most appropriate plane segment for each partition. However, in the absence of a ground plan or if it is not up to date, such methods become semi-automatic [4]. Rottensteiner et al. [2] presented an improved algorithm for automatic generation of 3D building models from high resolution aerial imagery and the LIDAR DSM (Digital Surface Model). Khoshelham et al. [5] applied a split-and-merge technique for automatic reconstruction of 3D roof planes. Park et al. [6] automatically re- 


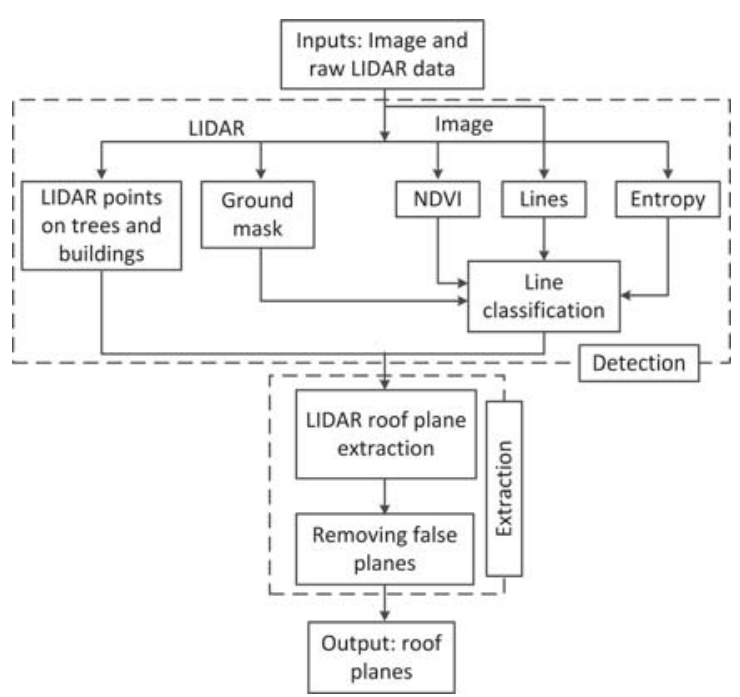

Fig. 1. Proposed 3D extraction of building roofs.

constructed large complex buildings using LIDAR data and digital maps. Dorninger and Pfeifer [4] proposed an automated method using LIDAR point clouds. Sampath and Shan [7] presented a solution framework for segmentation and reconstruction of polyhedral building roofs from high density LIDAR data. Habib et al. [8] generated polyhedral building models through integration of LIDAR data and stereo imagery. Cheng et al. [1] integrated multi-view aerial imagery with LIDAR data for 3D building reconstruction.

This paper proposes a new approach for automatic 3D roof extraction through an effective integration of LIDAR data and multispectral imagery. The LIDAR data is divided into two groups: ground and non-ground points. The ground points are segmented to generate a 'ground mask'. The nonground points are used to extract roof planes. The structural image lines are classified into several classes ('ground', 'tree', 'roof edge' and 'roof ridge') using the ground mask, colour orthoimagery and image texture information. In an iterative procedure, the non-ground LIDAR points near to a long roof edge or ridge line (known as the baseline) are used to obtain a roof plane. Promising experimental results are provided for 3D extraction of building roofs.

\section{PROPOSED EXTRACTION METHOD}

Fig. 1 shows an overview of the proposed building roof extraction procedure. The input data consist of raw LIDAR data, a DEM (Digital Elevation Model) and multispectral orthoimagery. The DEM is only used for an estimation of the ground height while generating the mask from the raw LIDAR data. In the detection step (top dotted rectangle in Fig. 1), the LIDAR points on the buildings and trees are separated as nonground points. The NDVI (Normalised Difference Vegetation

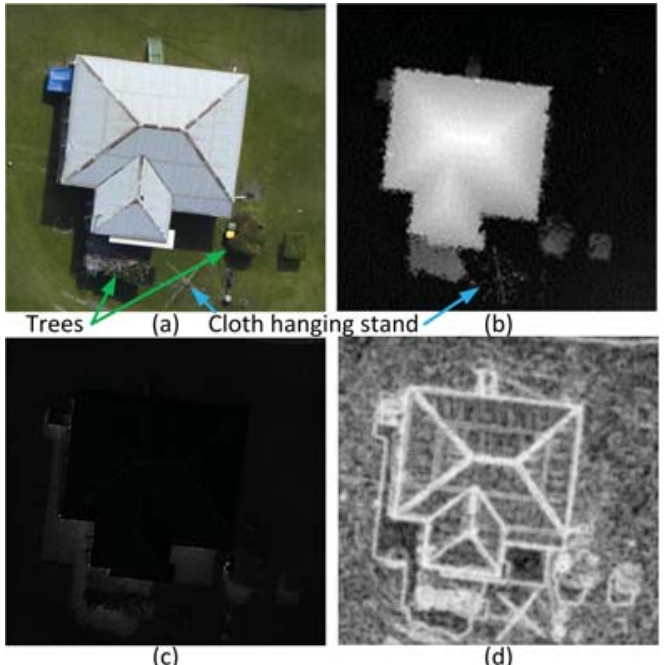

Fig. 2. A sample of a test scene: (a) RGB orthoimage, (b) LIDAR data shown as a grey-scale grid, (c) NDVI image from (a), and (d) entropy image from (a).

Index) is calculated for each image pixel location using the colour orthoimage. The texture information is estimated at each image pixel location using a grey-scale version of the image. The same grey level image is used to find the image lines that are at least $1 \mathrm{~m}$ in length. These lines are classified into several classes, namely, 'ground', 'tree', 'roof edge' and 'roof ridge'. In the extraction step (bottom dotted rectangle in Fig. 1), lines classified as roof edges and ridges are processed along with the non-ground LIDAR points. In an iterative procedure, the first plane is constructed using the longest edge or ridge line as a baseline. The next plane is constructed using the next longest line as a baseline, and so on. The image lines which are parallel or perpendicular to a baseline can be used as baselines on a priority basis to extract other neighboring planes first. While extracting a plane using a baseline, the neighbouring LIDAR points on a side of the baseline are used to define a robust seed region. The neighbouring plane is then iteratively grown to complete the whole plane.

Fig. 2a presents a sample scene of a test data set. It will be used to illustrate the different steps of the proposed extraction method. This scene is from Aitkenvale, QLD, Australia. Available data comprised of the first-pulse LIDAR data with a point spacing of $0.17 \mathrm{~m}$ (Fig. 2b), a DEM (with $1 \mathrm{~m}$ spacing), and an RGB colour orthoimage with a resolution of $0.05 \mathrm{~m}$. The original test scene is of size $2136 \times 1582$ pixels. Since there were shadows in the orthoimage, the NDVI image, shown in Fig. 2c, did not provide as much information as expected. Therefore, texture information in the form of entropy [9] (see Fig. 2d) is also employed based on the observation that trees are rich in texture as compared to building roofs. While a high entropy value at an image pixel indicates a texture (tree) pixel, a low entropy value indicates a 'flat' 


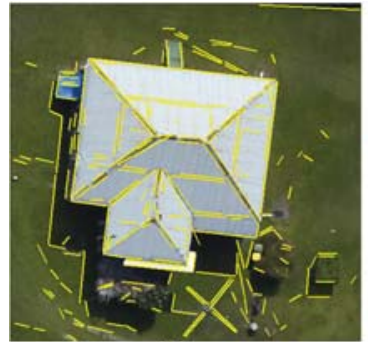

(a)

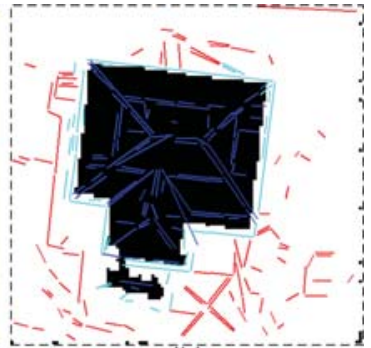

(b)
Fig. 3. Image line classification: (a) all extracted lines and (b) classified lines on ground mask. (Classes: green: 'tree', red: 'ground', cyan: 'roof edge' and blue: 'roof ridge'.)

(building roof) pixel. The entropy and NDVI information together will be used to classify roof edges and tree edges while classifying image lines. However, like the building detection algorithm in [9], the proposed extraction algorithm does not remove all trees at the initial stage. It employs a new procedure to remove false positive planes on trees.

\subsection{Finding Building Lines}

In order to extract lines from a grey-scale orthoimage, edges are first detected using the Canny edge detector. Corners are then detected on the extracted curves using a corner detector. On each edge, all the pixels between two corners or a corner and an endpoint, or two endpoints when enough corners are not available, are considered to form a separate line segment. If a line segment is less than $1 \mathrm{~m}$ in length it is removed. Thus trees having small horizontal areas are removed. Finally, a least-squares straight-line fitting technique is applied to properly align each of the remaining line segments. Fig. 3a shows the extracted lines from the sample test scene.

For each LIDAR point, the corresponding DEM height is used as the ground height $H_{g}$. If there is no corresponding DEM height for a given LIDAR point, the average DEM height in the neighbourhood is used as $H_{g}$. A height threshold $T_{h}=H_{g}+2.5 \mathrm{~m}$ [2] is applied to the raw LIDAR height. Consequently, the LIDAR data are divided into two groups: ground points which reflect from the low height objects such as ground, road furniture, cars and bushes, and non-ground points which reflect from elevated objects such as buildings and trees. The ground mask $M_{g}$, shown in Fig. 3b, is generated from the ground points following the procedure in $[9,10]$.

Three types of information are required to classify the extracted image lines into 'ground', 'trees', 'roof ridge' and 'roof edge': ground mask (Fig. 3b), NDVI (Fig. 2c) and the entropy mask [9]. While the NDVI image is derived from the RGBI orthoimage, the pseudo-NDVI image is derived from the RGB orthoimage if the infra-red band is not available [10]. In order to derive the the entropy mask, the entropy image shown in Fig. 2d is first estimated. Then the mask is created applying an entropy threshold (see [9] for more details).

For classification of the extracted image lines, a rectangular area of width $w_{d}=\frac{W_{m}}{2}$ on each side of a line is considered, where $W_{m}=3 \mathrm{~m}$ is assumed to be the minimum building width. A detailed procedure of this rectangular neighborhood setup can be found in [10]. In each rectangle, the percentage $\Phi$ of black pixels from $M_{g}$, the average NDVI (or pseudo-NDVI) value $\Upsilon$ and the percentage $\Psi$ of white pixels in the entropy mask are estimated. A binary flag $F_{b}$ for each rectangle is also estimated, where $F_{b}=1$ indicates that there are continuous black pixels in $M_{g}$ along the line.

For a line, if $\Phi<10 \%$ on both of its sides, then the line is classified as 'ground'. Otherwise, $\Upsilon$ and $\Psi$ are considered for each side where $\Phi \geq 10 \%$. If $\Upsilon>10$ for RGBI image or $\Upsilon>48$ for RGB image and $\Psi>30 \%$ on either of the sides then the line is classified as 'tree'. If $\Upsilon \leq 10$, or if $\Upsilon>10$ but $\Psi \leq 30 \%$, then the line is classified as 'roof ridge' if $F_{b}=1$ on both sides. However, if $F_{b}=1$ on one side only then it is classified as 'roof edge'. Otherwise, the line is classified as 'ground' ( $F_{b}=0$ on both sides), for example, for road sides with trees on the nature strip. The setup of all parameter values above have been adopted from [9, 10]. Fig. 3b shows different classes of the extracted image lines overlaid on the ground mask.

For each extracted line, its two end points and slope information are recorded to use in the extraction step described below. For roof edge and ridge lines, a point $P_{i}$ is also recorded which indicates the side of the line where $\Phi$ value is higher than the other side [10]. As shown in Fig. 4a, $P_{i}$ is defined with respect to the mid-point $P_{m}$ of an edge or ridge line when $\left|P_{i} . P_{m}\right|=w_{d}$. For a roof edge $P_{i}$ is on the corresponding roof plane and thus identifies the building side. For a roof ridge $P_{i}$ also helps finding the other side of the line, ie, the other roof plane.

\subsection{Constructing Roof Planes}

The following parameters will frequently be used throughout the extraction procedure. First, the flight distance $d_{f}$, which is the distance between two neighbouring LIDAR points, indicates the LIDAR point density. Second, the flat height threshold $T_{f}$ which is related to the error between the height of a LIDAR point and its actual height. Ideally, two points on a real flat (horizontal) plane should have the same heights, but there may be some error in their estimated heights by the LIDAR system. The parameter $T_{f}$ indicates this error and is set at $0.1 \mathrm{~m}$ in this study. Finally, a point that fits a plane should be within a plane threshold $T_{p}=0.15 \mathrm{~m}$ away.

Starting from the longest edge or ridge line (cyan coloured line in Fig. 4a), LIDAR points within a rectangle (of width $d_{f}$ on both sides of the line, as shown in magenta coloured line in Fig. 4a) are first examined. The points which have already been decided for any of the previously estimated planes are 


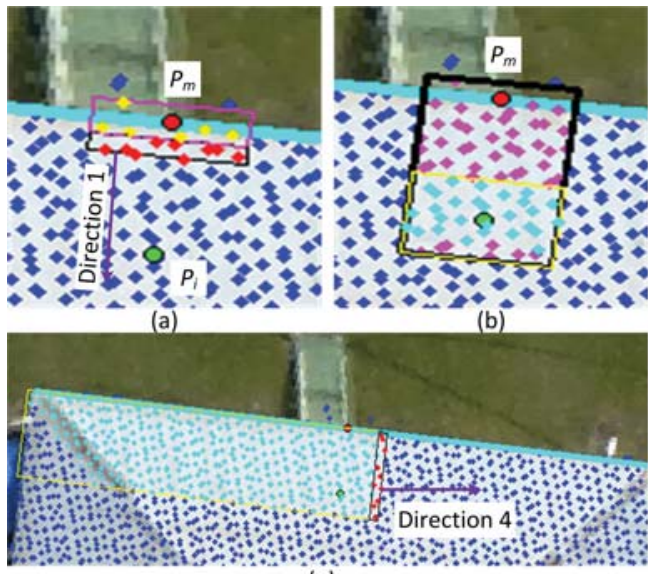

(e)

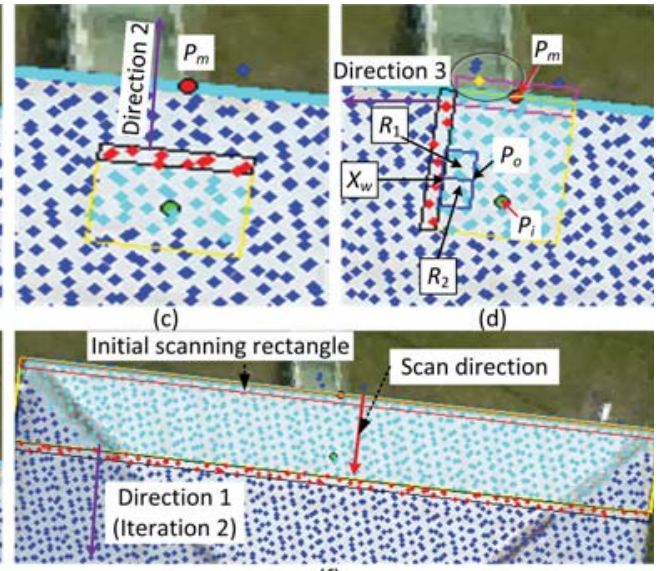

(f)

Fig. 4. Iterative extraction of a plane (coloured dots are LIDAR points, cyan coloured line is baseline, purple coloured arrows indicate directions for plane extensions): (a) initial plane around the baseline (yellow coloured dots inside magenta coloured rectangle form initial plane) and extension of initial plane (red dots inside black coloured rectangle are new candidates), (b) choosing stable seed region (LIDAR points) inside plane (cyan and magenta coloured points inside black coloured rectangle are now on plane, but cyan coloured points inside yellow coloured rectangle are chosen to restart plane growing), (c) extension of initial stable plane towards baseline, (d) extension of plane towards left, (e) extension of plane towards right and (f) plane after first iteration. In (a)-(f), red coloured dots inside black coloured rectangle are new candidates.

removed. The points which reflect from the wall are not considered too. The number of such points is small and their heights are low while compared to the actual points on the roof. A point within the rectangle is considered to be reflected from the wall if it has high height difference (more than $T_{f}$ ) with majority of the points within the rectangle. The region is extended towards Direction 1 considering a new rectangle (black coloured rectangle in Fig. 4a) until at least 4 suitable LIDAR points are obtained to solve the plane equation

$$
A x+B y+C z+D=0 .
$$

Then the region extension is continued towards Direction 1 until at least 1 point in the extended rectangle is compatible with the plane. The compatible points should not be used by a previous plane, their distances to the plane should be low (within $T_{p}$ ) and they should have similar heights (within $T_{f}$ ) with their neighbours. For a large plane a more robust seed region can be defined at this moment by stopping the plane extension in Direction 1 when it reaches a distance $1+2 d_{f} \mathrm{~m}$. The LIDAR points within the rectangle of width $2 d_{f}$ (cyan coloured points in Fig. 4b) now initiate the plane equation and the plane is now extended towards Direction 2 (see Fig. 4c) using the compatibility tests discussed above. Fig. $4 d$ shows all the compatible points in cyan colour, while the blue points within the black circle are now found to be incompatible. These points probably reflected from the nearby wall. As a result, the plane equation is more stable at this point.

The plane is then extended towards Directions 3 and 4 (Figs. 4d-e) respectively. The neighbourhood of a new point $X_{w}$ (shown in red colour) is a rectangular area of width $d_{f}$ and length $2 d_{f}$ (Rectangles $R_{1}$ and $R_{2}$ in Fig. $4 \mathrm{~d}$ ). At the end of extension in Direction 4, one iteration of the plane growing finishes, and the next iteration starts in Direction 1 as shown in Fig. 4f, ie, the extension of four sides of the plane continues iteratively. If no side is extended in an iteration, the iterative procedure stops. For example, the extraction of the current plane of the sample data set is completed in the second iteration and it is shown in Fig. 5a.

All the image lines which reside within an extended plane rectangle (black coloured rectangle in Fig. 5a, whose width and height of the plane rectangle are increased $1 \mathrm{~m}$ on each side) and either parallel or perpendicular to the previous baseline are kept into a queue $Q$ for priority processing. There were 21 such lines which are shown in yellow colour in Fig. 5a. Lines in $Q$ are processed first starting with the longest line first. Consequently, the extraction of a next plane is started with a new baseline shown as a thick yellow coloured line in Fig. 5a. If $Q$ is empty then other image edge and ridge lines are used as baselines. Fig. 5b shows all the extracted planes for the sample data set.

Finally, false planes constructed on trees are removed. Such planes are small in size and randomly oriented. Assuming that the minimum building width is $3 \mathrm{~m}$ [10], if the area of a plane or a group of neighbouring planes is smaller than $9 \mathrm{~m}^{2}$, the plane or the group of planes are removed. Furthermore, in order to find randomly oriented plane a point test is carried out for each extracted plane. A set of $2 \mathrm{D}$ random points is generated within the vicinity of the plane and for each point its height is estimated using the plane equation. If the estimated height of a randomly generated point is too high or too 


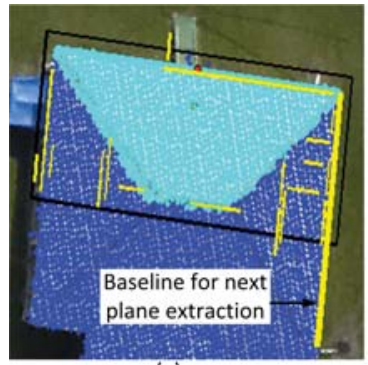

(a)

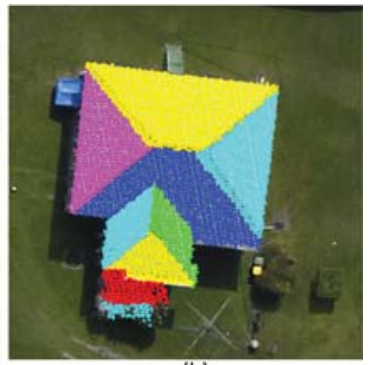

(b)
Fig. 5. Results of iterative plane extraction: (a) first plane and (b) all planes.

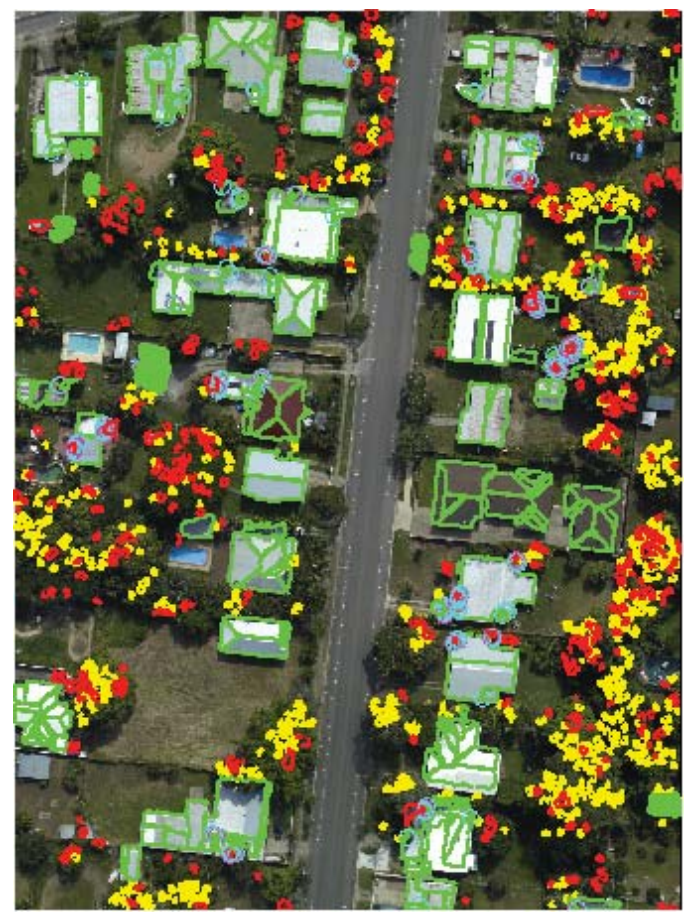

Fig. 6. Results on the first scene of the Aitkenvale data set. Green: extracted roof planes, other colours: false planes mostly extracted on trees and therefore removed.

low while compared to the LIDAR points on the plane, then the plane is decided to be a false plane.

\section{RESULTS AND DISCUSSIONS}

The test data sets employed cover two suburban areas in QLD, Australia: Aitkenvale (AV) and Harvey Bay (HB). There are two scenes available in the AV data set. The first scene (AV1) covers an area of $108 \mathrm{~m} \times 80 \mathrm{~m}$ and contains 58 buildings comprising 204 roof planes. The second scene (AV2) covers an area of $66 \mathrm{~m} \times 52 \mathrm{~m}$ and contains 5 buildings comprising 25 roof planes. The HB data set has one scene that covers
$108 \mathrm{~m} \times 104 \mathrm{~m}$ and contains 25 buildings consisting of 152 roof planes. Both of the data sets contain mostly residential buildings and can be characterized as outer suburban with medium housing density and moderate tree coverage that partially covers buildings. In terms of topography, while Aitkenvale is almost a flat area, Harvey Bay is somewhat hilly. Two dimensional reference data sets were created by monoscopic image measurement. All visible roof planes were digitized as polygons irrespective of their size. The reference data included garden sheds, garages, etc. These were sometimes as small as $1 \mathrm{~m}^{2}$ in area. Then both object- and pixel-based completeness, correctness and quality were estimated following an automatic and threshold free evaluation system [10]. While in object-based evaluation the number of planes is considered, in pixel-based evaluation the number of pixels is considered.

Fig. 6 shows the extracted planes on the AV1 scene. It is observed that almost all the roof planes are correctly extracted and false planes on trees are removed. However, due to nearby trees or small dormers on the roof there are a few cases where over segmentation causes some of the true planes to be extracted in two or more small components. Similar results were found in other two scenes.

On average, the object-based completeness, correctness and quality were $99.2 \%, 99.5 \%$ and $98.5 \%$ respectively. The pixel-based completeness, correctness and quality were $92.6 \%, 95.6 \%$ and $88.9 \%$ respectively. These numbers imply that the proposed roof extraction algorithm achieves a high level of accuracy.

Among the existing methods, Khoshelham et al. [5] evaluated results on 4 simple gable roofs having a total of 10 planes and showed object-based completeness $C_{m}$ and correctness $C_{r}$ of $100 \%$ and $91 \%$ respectively. The semiautomatic method by Habib et al. [8] offered $C_{m}=75 \%$ and $C_{r}=94 \%$ on an experimentation using 23 buildings comprising of 180 planes. Nevertheless, it could not construct planes which are less than $9 \mathrm{~m}^{2}$ in area. Jochem et al. [11] evaluated their LIDAR-based roof plane segmentation method on a large data set of 1003 roof planes and achieved $C_{m}=94.4 \%$ and $C_{r}=88.4 \%$. However, as the authors mentioned their method had the following shortcomings. Firstly, it could not construct planes of less than $6 \mathrm{~m}^{2}$ in area. Secondly, it could not remove very dense vegetation where the slope-adaptive LIDAR echo ratio is high. Moreover, it lost accuracy of the extracted planes as it used the interpolated LIDAR data. It is not clear from the experimental results whether the two methods in $[5,8]$ can work in vegetation having the similar heights as the neighbouring buildings and whether the method in [5] can extract small roof planes.

While the proposed algorithm in this paper is compared with the aforementioned three existing methods, like [5] and [11] the proposed method is fully automatic, while the method in [8] is a semi-automatic as it requires manual monoplotting procedure to delete incorrect boundaries and add necessary boundary segments. The two methods in $[5,8]$ might 
offer better planimetric accuracy than the proposed and the method in [11] due to use of image lines to describe the plane boundaries. However, the proposed method may offer better vertical accuracy than the existing three methods as it uses the raw LIDAR data to describe the planes.

The evaluation results presented in this paper are on 88 buildings consisting of 381 roof planes. The proposed method can extract an individual planes as small as $1 \mathrm{~m}^{2}$ and apply a new rule-based procedure to remove all kinds of vegetation. In terms of object-based completeness and correctness, the proposed method offered higher performance $\left(C_{m}=99.2 \%\right.$ and $C_{r}=99.5 \%$ ) than three existing methods. Moreover, all of the existing methods did not show results using the pixelbased evaluation metrics. In contrast, the proposed method showed high performance in pixel-based evaluation as well.

\section{CONCLUSION AND FUTURE WORK}

This paper has presented a new method for automatic 3D roof extraction through an effective integration of LIDAR data and multispectral orthoimagery. The LIDAR points are divided into two groups: ground and non-ground points. The ground points are exploited to constitute the 'ground mask'. The structural image lines have been extracted from the greyscale version of the orthoimage and then classified into several classes such as 'ground', 'tree', 'roof edge' and 'roof ridge' using the ground mask, the NDVI image and the entropy image, which are generated from the input data. The lines from the later two classes are used to fit roof planes to the neighbouring non-ground LIDAR points.

While compared with the three existing methods, the proposed method can extract planes as small as $1 \mathrm{~m}^{2}$ in area and can work in the presence of dense vegetation. The proposed method is fully automatic and experimental results show that it offers high extraction rates.

Future work includes rectification of over and under segmentation cases and testing the algorithm on more complex data sets. In addition, it will be interesting to test the algorithm on real data with low LIDAR point density.

\section{Acknowledgment}

Dr. Awrangjeb is the recipient of the Discovery Early Career Researcher Award by the Australian Research Council (project number DE120101778). The authors would like to thank Ergon Energy (www.ergon.com.au) for providing the data sets.

\section{REFERENCES}

[1] L. Cheng, J. Gong, M. Li, and Y. Liu, "3d building model reconstruction from multi-view aerial imagery and lidar data," Photogrammetric Engineering and Remote Sensing, vol. 77, no. 2, pp. 125-139, 2011.

[2] F. Rottensteiner, J. Trinder, S. Clode, and K. Kubik, "Fusing airborne laser scanner data and aerial imagery for the automatic extraction of buildings in densely built-up areas," in Proc. ISPRS Twentieth Annual Congress, Istanbul, Turkey, 2004, pp. 512-517.

[3] G. Vosselman and S. Dijkman, "3d building model reconstruction from point clouds and ground plans," International Archives of the Photogrammetry and Remote Sensing, vol. XXXIV, no. 3/W4, pp. 37-44, 2001.

[4] P. Dorninger and N. Pfeifer, "A comprehensive automated $3 \mathrm{~d}$ approach for building extraction, reconstruction, and regularization from airborne laser scanning point clouds," Sensors, vol. 8, no. 11, pp. 7323-7343, 2008 .

[5] K. Khoshelham, Z. Li, and B. King, "A split-andmerge technique for automated reconstruction of roof planes," Photogrammetric Engineering and Remote Sensing, vol. 71, no. 7, pp. 855-862, 2005.

[6] J. Park, I. Y. Lee, Y. Choi, and Y. J. Lee, "Automatic extraction of large complex buildings using lidar data and digital maps," International Archives of the Photogrammetry and Remote Sensing, vol. XXXVI, no. 3/W4, pp. 148-154, 2006.

[7] A. Sampath and J. Shan, "Segmentation and reconstruction of polyhedral building roofs from aerial lidar point clouds," IEEE Transactions on Geoscience and Remote Sensing, vol. 48, no. 3, pp. 1554-1567, 2010.

[8] A. F. Habib, R. Zhai, and K. Changjae, "Generation of complex polyhedral building models by integrating stereo-aerial imagery and lidar data," Photogrammetric engineering and remote sensing, vol. 76, no. 5, pp. 609$623,2010$.

[9] M. Awrangjeb, C. Zhang, and C. S. Fraser, "Building detection in complex scenes thorough effective separation of buildings from trees," ASPRS Journal of Photogrammetric Engineering \& Remote Sensing, vol. 78, no. 7, pp. 729-745, 2012.

[10] M. Awrangjeb, M. Ravanbakhsh, and C. S. Fraser, "Automatic detection of residential buildings using lidar data and multispectral imagery," ISPRS Journal of Photogrammetry and Remote Sensing, vol. 65, no. 5, pp. 457-467, 2010.

[11] A. Jochem, B. Hfle, V. Wichmann, M. Rutzinger, and A. Zipf, "Area-wide roof plane segmentation in airborne lidar point clouds," Computers, Environment and Urban Systems, vol. 36, no. 1, pp. 54-64, 2012. 